\title{
«Ich lebe für die Perfektion»
}

\section{Daniel Lüthi}

Freier Journalist und Fotograf, Medientrainer, Bern

Tag der offenen Tür: 16. April 2016, 10-16 Uhr
Da und dort stehen letzte Zügelkisten, einige Räume sind noch leer. Bald aber wird der 50-Millionen-Bau eingeweiht. " «Wir platzten aus allen Nähten», sagt Michael Thiel. «Jetzt können wir auf einer nur wenig grösseren Fläche massiv mehr Leistung erbringen. Es ist das perfekte Gebäude für unsere Ansprüche.» Kein Zweifel: Die neue Augenklinik ist sein Stolz. Und «perfekt» deshalb das passende Attribut.

\section{Spielertrainer}

Die Anordnung der Räume sei jetzt auf die Bedürfnisse der Patienten und die Abläufe einer Augenklinik optimal abgestimmt, schwärmt Thiel, «früher gab es hier fast ausschliesslich Bettenzimmer, man war nicht auf ambulante Tätigkeiten ausgerichtet». Viel Licht, keine gefangenen Gänge, einladende Empfangs- und Wartezonen: Patienten könnten sich jetzt besser orientieren und auch Mitarbeitende würden sich im Neubau wohler fühlen. «Gerade für sie ist dieses Gebäude eine Wertschätzung. Gute Fussballer spielen auch gerne in einem schönen Stadion.» Der Vergleich mit dem Fussball kommt immer wieder.

Michael Thiel ist nicht nur Arzt, (zu etwa 60 Prozent, wie er sagt), sondern auch Chef. Vorgesetzter von rund 200 Mitarbeitenden in der Augenklinik und knapp 800 Angestellten im Departement Spezialkliniken. Dazu gehören das Frauenspital, die Anästhesie, die Intensivstation und die Rettungsdienste. «Ich bin Spielertrainer», sagt Thiel, «also ein Trainer, der mitspielt - und

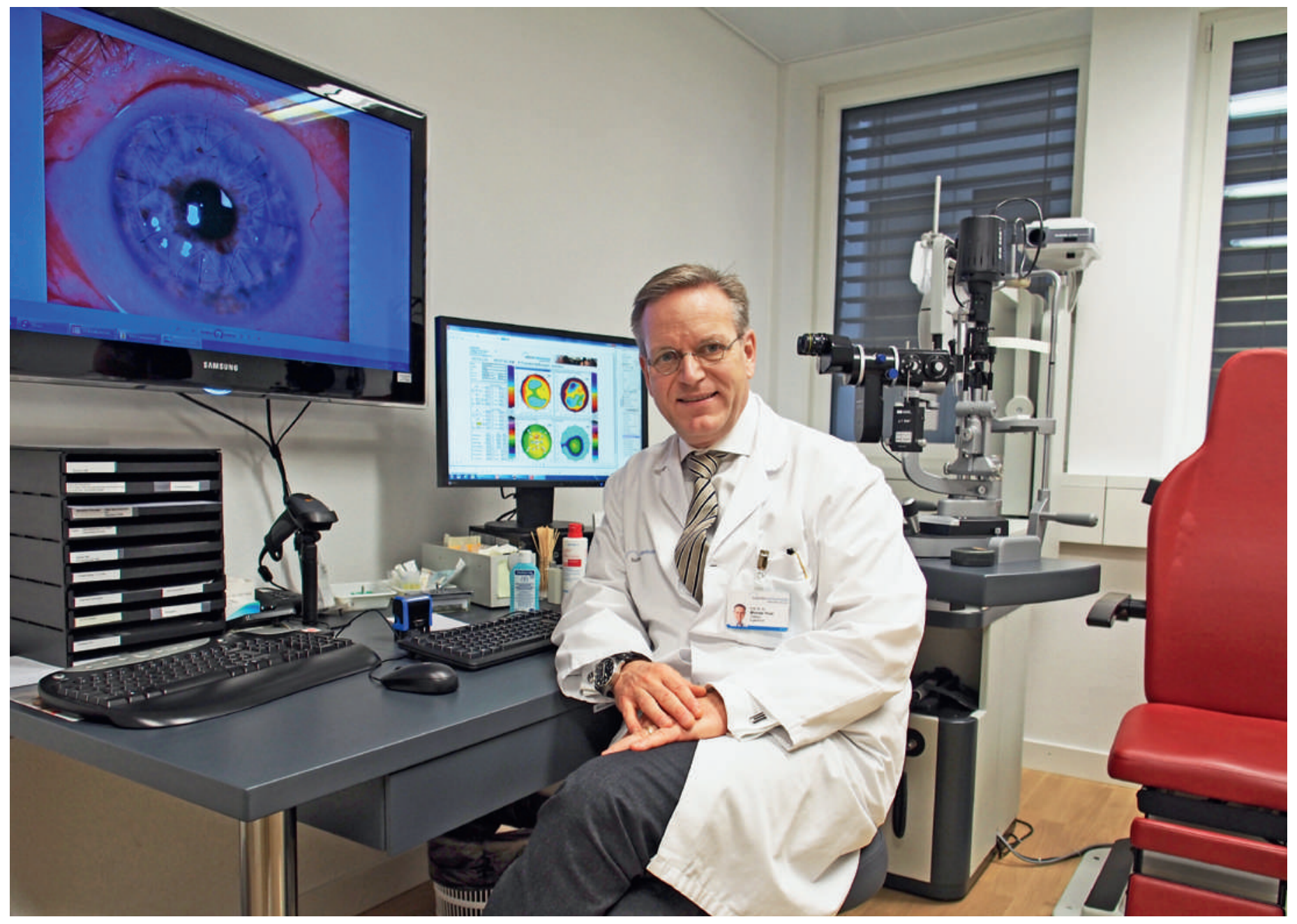


zwar nicht bloss an der Seitenlinie. Ich nehme Pässe $\mathrm{ab}$ und spiele sie weiter. Zum Beispiel dann, wenn die Frauenklinik in der Stadt eine Hebammenpraxis eröffnen will. Mein Team soll sich optimal entfalten können. Deshalb sehe ich mich nicht primär als Vorgesetzter, sondern als Interessenvertreter und Coach. Jeder soll aus seinem Potential das Optimum machen können.» Wie sehe ich mich? Und wie sehe ich die anderen? Bei einem Augenarzt haben diese Fragen eine spezielle Bedeutung.

\section{Hornhaut-Spezialist}

«Ich sehe auch Dinge, die man mit den Augen nicht sehen kann", sagt Thiel. Eine Landkarte zum Beispiel könne er einfach als dreidimensionale Landschaft wahrnehmen. «Das hilft mir beim Arbeiten. Denn ich habe auf sehr kleinem Raum mit sehr vielen kleinen, anfälligen, verletzlichen Strukturen zu tun. Da muss ich sehr präzise sein können.» Das ist der technische, mechanische Aspekt seiner ärztlichen Tätigkeit. «Mikro-manuelle Arbeiten haben mich schon als Kind fasziniert, und noch heute kann ich eine enthusiastische Freude dafür entwickeln.»

Michael Thiels Spezialgebiet ist die Hornhaut, «das klare Fenster vorne am Auge.» In seiner Klinik werden etwas mehr als die Hälfte aller HornhautTransplantationen in der Deutschschweiz gemacht. 2015 waren es 242 Hornhauttransplantationen, insgesamt wurden hier im vergangenen Jahr über 7000 komplexe chirurgische Eingriffe am Auge durchgeführt. Transplantationen sind nur möglich dank Organ- bzw. Gewebespenden. «Die Spendefreudigkeit der Schweizer Bevölkerung ist leider sehr beschränkt», sagt Thiel dazu. «Man ist lieber Empfänger als Spender. Die Wahrscheinlichkeit, etwas zu benötigen, ist übrigens vierzehnmal grösser als jene, überhaupt etwas geben zu können.» Der Vorteil einer Hornhaut sei, dass man sie bis vier Wochen lang aufbewahren kann. «Und dass sie für den wartenden Patienten oft nicht so vital ist wie beispielsweise ein Herz oder eine Niere.»

Selbstverständlich habe er persönlich schon lange «ja» gesagt zur Organspende. Der Spenderausweis, den er als Beweis aus seinem Portemonnaie fischt, ist 1995 ausgestellt worden.

\section{Sehen und wahrnehmen}

Was kann Thiel erreichen mit seinem Handwerk, seiner Kunst? «Jemand, der schlecht sieht, wird wieder klarer sehen. Sein Bild wird von trüb und neblig wieder scharf. Vor allem aber: Seine Lebensqualität wird besser.»

\section{Michael Thiel}

Prof. Dr. med. Dr. phil. Michael Thie wurde 1964 in Zürich geboren. Er studierte Medizin an der Universität Basel, wo er 1991 zum Dr. med. promovierte. Am UniversitätsSpital Zürich USZ bildete er sich 1993 bis 97 zum Augenarzt weiter. 1997 bis 2000 folgte ein Forschungsaufenthalt im australischen

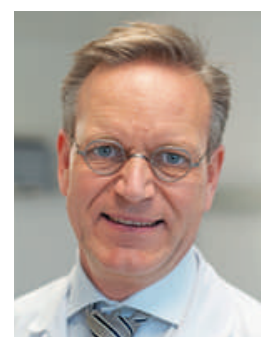

Adelaide mit einer zweiten Promotion zum Dr. phil. auf dem Gebiet der Transplantationsimmunologie. Dort spezialisierte er sich auch auf Hornhauterkrankungen und -transplantationen. Im Jahr 2000 kehrte er ans USZ zurück. 2005 habilitierte er in Zürich, 2007 wechselte er ans Luzerner Kantonsspital als Chefarzt der Augenklinik. Seit 2012 ist er dort Leiter des Departements Spezialkliniken und Mitglied der Geschäftsleitung. 2013 ernannte ihn die Universität Zürich zum Titularprofessor für Ophthalmologie. Michael Thiel ist verheiratet und Vater von zwei Söhnen. Er lebt in Birchwil bei Zürich und in Luzern.

Mit anderen Worten: Thiel sieht als erstrebenswertes Resultat seiner Arbeit weit mehr als Änderungen an einem Gewebe und Verbesserungen in einem Organ. «Es geht nicht nur darum, ein isoliertes Problem zu sehen, sondern den Patienten als ganzen Menschen mit

\section{"Jeder soll aus seinem Potential das Optimum} machen können.»

seinen Bedürfnissen wahrzunehmen.» Wiederum ein spezieller Punkt, gerade bei einem Augenarzt: der wesentliche Unterschied zwischen «sehen" und «wahrnehmen». «Sehen ist bloss ein Signal», erklärt Thiel. «Wahrnehmen ist weit mehr.» Bezogen auf die Ophthalmologie als spitzenmedizinische Disziplin: «Wenn ein Spezialist seinen Blick ausschliesslich auf die rund drei Kubikzentimeter fokussiert, die seine Spezialisierung ausmachen, dann wird dies zum Damoklesschwert. Dann degradiert er sein Spezialistentum zur rein technischen Eitelkeit.» Immer gehe es auch darum, Wunschdenken und reale Optionen gegeneinander abzuwägen «zu beurteilen, nicht einfach etwas zu machen».

\section{Aufwand und Ertrag}

Zurück zum Fussball: Wer in der obersten Liga spielt und dort bleiben will, muss kontinuierlich Besonderes leisten. Und viel. 75-Stunden-Wochen sind bei Michael Thiel die Regel, dreimal pro Woche übernachtet er in Luzern, also nicht bei seiner Familie. «Ich komme aus einer Generation, für die ein solches Pensum normal ist», kommentiert er, und ja, «meine Frau muss privat einiges ausbaden, ohne sie hätte ich das alles nie geschafft. Aber ich will mir diesen Zeiteinsatz leisten - ich 
lebe für die Perfektion. Mein persönlicher Aufwand dafür ist mir gleich.» Und was sagt er einem Assistenten, der sich auf seine vertraglich zugesicherte 50-StundenWoche beruft? «Ich mag sie ihm gönnen. Aber ich mache mir Sorgen um die Qualität. Begabte Nachwuchskräfte werden gebremst, ja entmündigt, wenn sie durch die Stempeluhr zu reinen Zeitarbeitern degradiert werden. Die rigide Anwendung des geltenden Arbeitszeitgesetzes hemmt ihre Kreativität und die Befriedigung ihres Wissensdurstes.» Die 50-Stundenwoche als Jahresdurchschnitt wäre kein Problem, sagt er, es gehe um die rigide Anwendung der Zeitgrenze in jeder einzelnen Woche: «Es tut mir weh, wenn jemand vorzeitig den Operationssaal oder eine Fallbesprechung verlassen muss, weil sein Zeitguthaben abgelaufen ist.» In der Fussballsprache: «Einem Spitzenspieler sagt man ja auch nicht, er dürfe nur einmal pro Woche trainieren. Unser Gesundheitssystem lebt davon, dass Ärzte manchmal überdurchschnittliche Leistungen erbringen.»

\section{«Sehen ist bloss ein Signal.»}

Sprechen wir doch gleich auch über das zweite unangenehme Thema, das mit dem erwähnten Aufwand und Knowhow unmittelbar zusammenhängt: das hohe Einkommen, das Spezialisten immer wieder vorgeworfen wird. «Die sogenannten Spitzengehälter machen in einem Spital bei den Kosten den Unterschied nicht aus", sagt Thiel. Entscheidend jedoch seien die Einkommensunterschiede zwischen Spital und Praxis. «Wegen der hohen Gehälter in der Praxis ist es für uns oft schwierig, die Leute im Spital-Team zu behalten. Ansonsten jedoch ist die Geld-Frage oft eine reine Neid-Diskussion.»

\section{Laser als Lifestyle?}

Der Luzerner Augenklinik ist das Augenlaserzentrum Zentralschweiz angegliedert. Andernorts, in Bern oder Zürich zum Beispiel, hätten die öffentlichen Spitäler dieses Feld der Privatwirtschaft überlassen, sagt Thiel. «Die Diskussion um Laser-Operationen bei Kurz- oder Weitsichtigkeit, um die refraktive Chirurgie also, wird leider allzu oft abschätzig als reine Lifestyle-Diskussion geführt», bedauert er. "Dabei geht es nicht bloss um die Brillenfreiheit; es gibt Fehlsichtigkeiten, die den Alltag massiv beeinträchtigen.»

Thiel selber trägt eine Brille. Warum hat er seine Augen nicht lasern lassen? «Ich hab's mir immer wieder überlegt», antwortet er. "Das Risiko, dass das Resultat eines solchen Eingriffs nicht optimal ist, ist sehr, sehr klein. In meiner Tätigkeit als Mikrochirurg benötige ich aber zwei perfekte Augen für die Tiefenwahrnehmung. Daher würde meine chirurgische Arbeitsfähigkeit durch ein gutes, aber nicht perfektes Resultat an einem der beiden Augen bereits in Frage gestellt. Mein höchstes Gut ist die Qualität meiner Leistung, und dafür gehe ich kein noch so kleines Risiko ein, wenn es vermeidbar ist. Im Übrigen trage ich nicht ungern eine Brille.»

\section{Und sonst?}

In Thiels Büro steht auf dem Gestell hinter dem Pult das Modell der Segeljacht Alinghi. Das Schiff deutet darauf hin, dass es im Leben dieses Mannes trotz allem noch anderes gibt als den Beruf. Ja, er segle seit seinem 16. Lebensjahr, erzählt er. Ab und zu sei er beispielsweise im Mittelmeer unterwegs. Und er baue selber auch Boote, zur Zeit ein Holzkanu. Das komme seinem handwerklichen Interesse entgegen.

Grundsätzlich sagt er zum Thema «Work-Life-Balance»: «Mein Bedürfnis nach persönlicher Freizeit ist wohl eher bescheiden. Ich brauche für meine Befriedigung die Freizeit als Entschädigung nicht.» Und das Kanu, von dessen Bau er eben mit einem freudigen Glänzen in den Augen gesprochen hat? «Daran bin ich seit fünf Jahren», ergänzt er amüsiert. «Und das dauert noch lange. Das Problem ist halt, dass der Tag nur 24 Stunden hat.»

\section{Die nächste Begegnung mit ...}

Am Ende jeden Monats stellt die Schweizerische Ärztezeitung eine Persönlichkeit vor, die sich im Gesundheitswesen engagiert. Im April schildert Daniel Lüthi seine Begegnung mit Eva Gollwitzer, Biologin und Forscherin am CHUV in Lausanne. 